\title{
DEMONSTRATION OF A MICROFABRICATED SELF-OSCILLATING FLUIDIC HEAT ENGINE (SOFHE)
}

T. Monin ${ }^{1,2,3 *}$ A. Tessier-Poirier ${ }^{1}$, A. Amnache ${ }^{1}$, T. Skotnicki ${ }^{3}$, S. Monfray ${ }^{3}$, F. Formosa ${ }^{2}$ and L. G. Fréchette ${ }^{1}$

${ }^{1}$ Laboratoire Nanotechnologies et Nanosystèmes (LN2), Interdisciplinary Institute for Techn. Innovation (3IT), Université de Sherbrooke, Canada

${ }^{2}$ SYMME, Université Savoie Mont Blanc, Annecy-le-vieux, France

${ }^{3}$ STMicroelectronics, Crolles, France

We present the first microfabricated heat engine based on the self-induced oscillations of a liquid piston exposed to a constant heat source. The unique operating principle of this self-oscillating fluidic heat engine, or SOFHE, was previously introduced at the macroscale [1] as an approach to convert waste heat into electricity to power wireless sensors. Here, we have implemented the core principle in a glass microchip for the first time and demonstrated the self-oscillating behavior at microscale.

\section{NEED FOR THERMAL ENERGY HARVESTING}

By integrating sensors everywhere around us, the Internet of Things (IoT) promises to truly connect the physical and cyber worlds, without the need for human intervention. Distributed sensors will however require long lasting power sources, which would ideally be recharged perpetually from ambient energy. Heat is often available to power sensors, therefore low-cost and compact thermal energy harvesters could play a key role in realizing the IoT's potential. Other than thermoelectrics, various MEMS-based heat engines have been studied [2-4]. They typically require an actuated or bi-stable thermal switching mechanism to induce the cyclic behavior required to implement a thermodynamic cycle.

\section{OPERATING PRINCIPLE}

In our previous work [1], we proposed a new principle to create the cyclic motion from a steady heat source. It consists of creating a vapor bubble in a tube filled with liquid, by locally heating the tube (Fig 1). As the evaporation occurs, the bubble pressure increases, pushing on the surrounding liquid column acting as a liquid piston (LP). Once the bubble grows beyond the hot zone, the vapor condenses, reducing the bubble pressure and pulling the LP back into the hot zone. Given the inertia of the LP, it tends to overshoot the equilibrium position and enters further into the hot zone. This promotes evaporation, repeating the cycle. The system can be viewed as a spring-mass system, where the compressible vapor in the bubble acts as the spring and the LP as the mass (Fig 1). Phase change acts as a negative damping (or feedback) and leads to the start-up of the engine by making the equivalent mechanical oscillator unstable. From the start-up, the oscillating amplitude increases until the system reaches a steadystate limit cycle. Although we have studied this unique behavior experimentally and analytically [1,5], this work is the first to implement it on a chip, as a building block for thermal energy harvesters.

\section{SOFHE MICROFABRICATION AND RESULTS}

The microfabricated SOFHE consists of a liquid-filled microchannel with internal grooves, heated at one end and cooled at the other. The chip is made of glass to minimize heat conduction along the chip, which is detrimental for thermal efficiency and achievable temperature gradient. Based on our experience at macroscale, wicking structures in the channel were beneficial to facilitate the self-oscillating behavior, so the microchannel was formed by wet etching two half-channels of slightly different widths on separate chips that are then bonded to form the channel (Fig. 2). The overhang creates a sharp corner in the channel, acting as the desired wicking structure. Borosilicate glass is etched by $\mathrm{HF}(49 \%)$ with a Cr-Au-Kapton mask, allowing depths over 250 microns (Fig. 3). The wafers where then bonded by forming a eutectic mixture between Au on one wafer and PECVD amorphous silicon on the other wafer (Fig. 4).

The test apparatus is presented in Fig. 5, with the meniscus oscillations extracted from high-speed video (Fig. 6). The engine's self-induced oscillation frequency was $542 \mathrm{~Hz}$, with an amplitude of $>10$ microns. This is the first demonstration of the selfoscillating behavior on a chip, providing an initial building block for a new class of thermal energy harvesting MEMS.

\section{WORD COUNT: 598}

\section{REFERENCES}

[1] T. Monin, A. Tessier-Poirier, É. Léveillé, A. Juneau-Fecteau, T. Skotnicki, F. Formosa, S. Monfray, L.G. Fréchette, "First experimental demonstration of a self-oscillating fluidic heat engine (SOFHE) with piezoelectric power generation," J. of Phys.: Conf. Ser., vol. 773, no 012039, from 16th Int'1 Workshop on Micro and Nanotech. for Power Generat. and Energy Convers. Apps (PowerMEMS 2016), Paris, France, 69 Dec., (2016).

[2] S. Whalen, M. Thompson, D. Bahr, C. Richards, R. Richards, "Design, fabrication and testing of the P3 micro heat engine", Sensors and Actuators, Vol. 104, pp. 290-298, (2003).

[3] O. Puscasu, S. Monfray, C. Maitre, J.P. Permeant, F. Boeuf, G. Savelli, F. Gaillard, D. Guyomar, T. Skotnicki, "A disruptive technology for thermal to electrical energy conversion", Microelectronics Journal, Vol. 45, No. 5, pp. 554-558, (2014).

[4] T. Huesgen, J. Ruhhammer, G. Biancuzzi and P. Woias, "Detailed study of a micro heat engine for thermal energy harvesting", J. Micromech. Microeng. Vol. 20, (2010).

[5] A. Tessier-Poirier, T. Monin, É. Léveillé, F. Formosa, S. Monfray, L.G. Fréchette, "Influence of nonlinearities on the power output of the Self-Oscillating Fluidic Heat Engine (SOFHE), " J. of Phys.: Conf. Ser., vol. 773, no 012113, from 16th Int'l Workshop on Micro and Nanotech. for Power Generat. and Energy Convers. Apps (PowerMEMS 2016), Paris, France, 6-9 Dec., (2016).

\footnotetext{
${ }^{*}$ Currently at X-Fab, Corbeil-Essonnes, France
} 


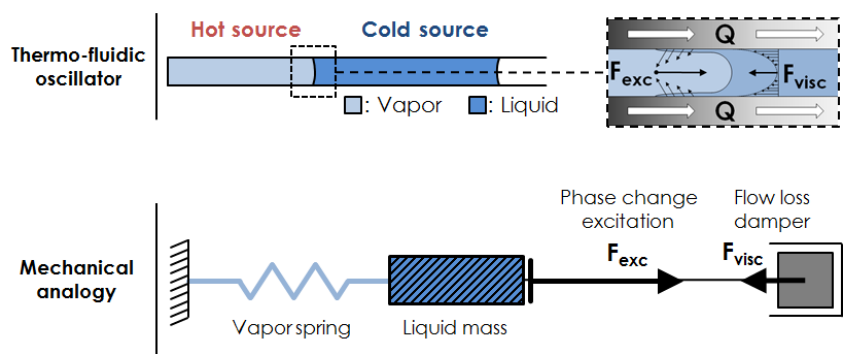

Figure 1: Schematic of the self-oscillating fluidic heat engine (SOFHE), with the spring-mass analogy.

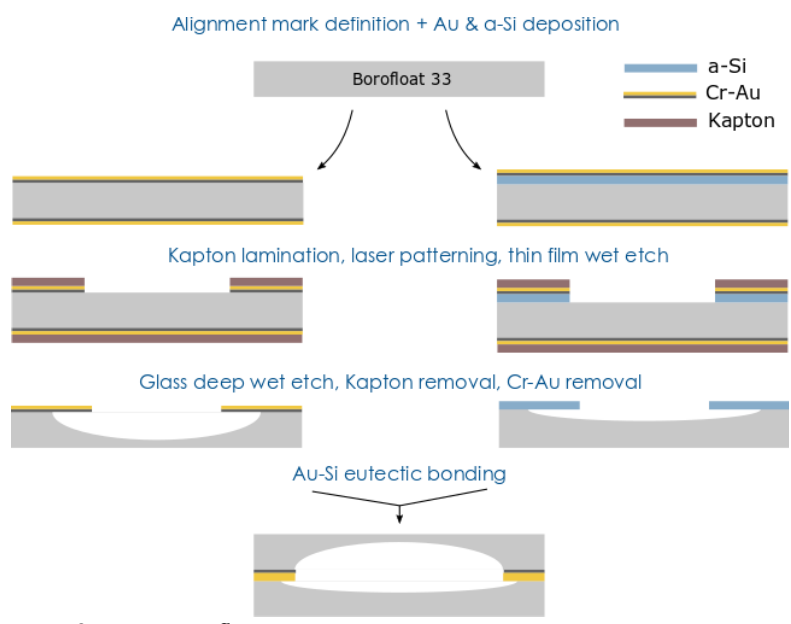

Figure 2: Process flow.

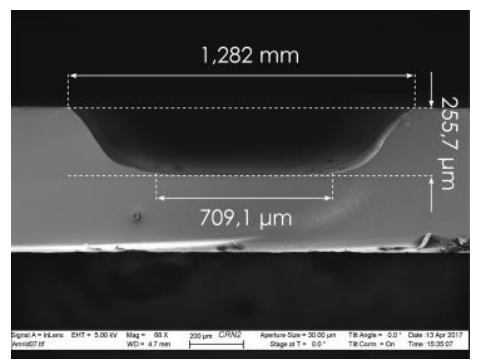

Figure 3: Wet etched half-microchannel in Borosilicate glass.
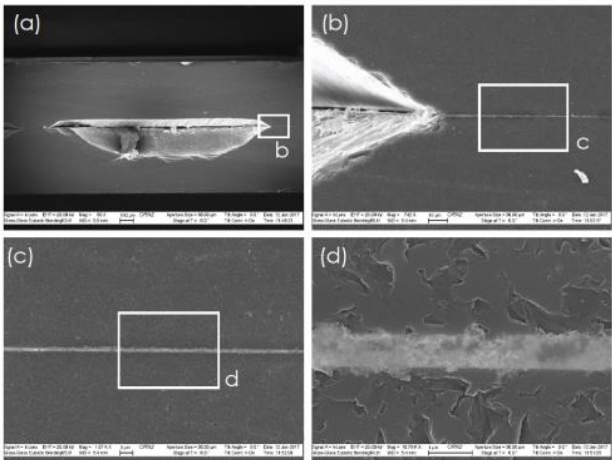

Figure 4: Device cross section after bonding, showing sharp corner formed for wicking purpose and the eutectic bonded interface using a thin PECVD a-Si layer and an Au layer to join the Borosilicate glass wafers.

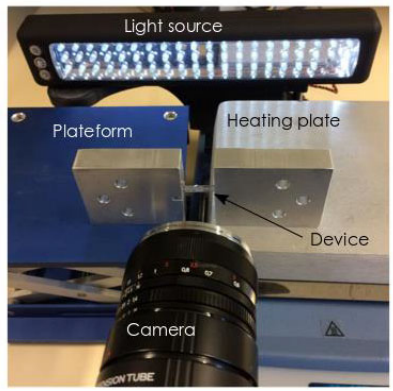

a) Test apparatus

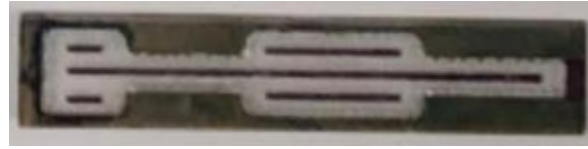

b) SOFHE microfluidic chip (6 x $25 \mathrm{~mm})$

Figure 5: Test apparatus (a), consisting of hot and cold plates supporting the chip at opposite ends, and a high-speed camera to visualize the meniscus position over time. Test chip close-up(b).

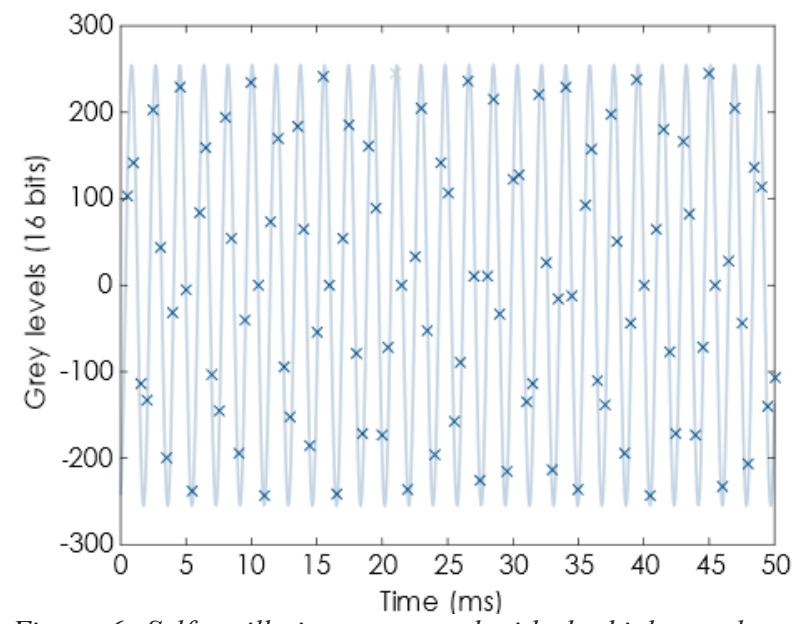

Figure 6: Self-oscillations measured with the high-speed camera achieved using water in the above SOFHE chip at a hot plate temperature of $175^{\circ} \mathrm{C}$.

Table 1: Measured operating conditions for the microfabricated SOFHE.

\begin{tabular}{|l|c|}
\hline \multicolumn{2}{|c|}{ Self-oscillating operation } \\
\hline Frequency & $542 \mathrm{~Hz}$ \\
\hline Amplitude & $>10 \mu \mathrm{m}$ \\
\hline
\end{tabular}

